\title{
Daurisoline Suppresses Growth of Esophageal Squamous Cell Carcinoma by Inhibiting MEK1/2 In Vitro and In Vivo
}

Donghao Wang

Zhengzhou University

Ning Yang

Zhengzhou University

Xiaofan Zhang

Zhengzhou University

Mingzhu Li

Zhengzhou University

Xin Li

Zhengzhou University

Lili Zhao

Zhengzhou University

Qiang Yuan

Zhengzhou University

Yin Yu

Zhengzhou University

Jing Lu

Zhengzhou University

Jimin Zhao

Zhengzhou University

Yanan Jiang

Zhengzhou University

Zigang Dong

Zhengzhou University

Kangdong Liu ( $\sim$ kdliu@zzu.edu.cn )

Zhengzhou University

\section{Research}

Keywords: ESCC, phosphoproteomics, daurisoline, MEK1/2, ERK1/2, proliferation, PDX 
Posted Date: May 7th, 2021

DOl: https://doi.org/10.21203/rs.3.rs-416241/v1

License: (c) (i) This work is licensed under a Creative Commons Attribution 4.0 International License. Read Full License 


\section{Abstract \\ Background}

Esophageal squamous cell carcinoma (ESCC) accounts for $90 \%$ of esophageal cancer and has a high mortality rate worldwide. The clinical treatment of ESCC is mainly surgical resection. The five-year survival rate of ESCC patients in developing countries is less than $20 \%$. Therefore, identifying new and effective drugs that can prevent the occurrence and recurrence of ESCC is clinically significant. Here, daurisoline, a bis-benzylisoquinoline alkaloid, was found to have an anticancer effect on ESCC.

\section{Methods}

We investigated the effects of daurisoline on ESCC cell growth and proliferation using ESCC cell lines (KYSE150 and KYSE450 cells) and tumor growth in an ESCC patient-derived xenograft model. Phosphoproteomics was used to identify changes in protein phosphorylation after daurisoline treatment. Molecular docking simulation, pull down assay and amino acid mutation experiments were conducted to determine the target proteins and specific amino acid binding sites of daurisoline. In vitro kinase assay was used to determine the effect of daurisoline on protein phosphorylation. The correlation between MEK1/2 and ERK1/2 expression levels in ESCC was analyzed using TCGA database.

\section{Results}

In vitro experiments showed that daurisoline inhibited the proliferation and anchorage-independent growth of ESCC cells. In vivo experiments indicated that daurisoline significantly inhibited tumor growth. Phosphoproteomics analysis revealed that daurisoline reduced ERK1/2 phosphorylation. A pull down assay showed that daurisoline could bind to MEK1/2. In vitro kinase assay confirmed that daurisoline inhibited the biological functions of MEK1/2. We observed a significant correlation between MEK1 and ERK2 in ESCC from the TCGA database.

\section{Conclusion}

Daurisoline is a MEK1/2 inhibitor that suppressed ESCC growth in vitro and in vivo.

\section{Background}

Esophageal cancer (ESCA) ranks seventh and sixth in incidence and mortality, respectively, among all cancers worldwide [1]. Esophageal squamous cell carcinoma (ESCC) and esophageal adenocarcinoma (EAC) are two main pathological classifications of ESCA [2]. It has been reported that $90 \%$ of ESCA cases are ESCC worldwide [3]. Clinical treatment of ESCC is mainly surgery, chemotherapy and radiotherapy, but the prognosis of ESCC is poor [4]. The five-year survival rate of ESCC patients in developing countries is 
less than $20 \%$ [5]. Therefore, it is of great clinical significance to find drugs that can prevent the occurrence and recurrence of ESCC [6].

Activation of the MEK1/2-ERK1/2 pathway plays an essential role in the proliferation, differentiation, invasion, and metastasis of cancer cells [7]. A large number of studies showed that the changes in the structure and expression level of MEK1/2 are closely related to the occurrence of tumors [8]. Therefore, MEK1/2 inhibitor is considered to be one of the promising areas of cancer research [9]. Currently, a variety of MEK $1 / 2$ inhibitors have been found, some of which have been used in the treatment of cancer. Selumetini is used to treat melanoma, and selumetinib is used to treat advanced differentiated thyroid cancer and plexus neurofibroma [10-11].

Screening natural compounds is an effective way to find anticancer drugs. Bis-benzylisoquinoline alkaloids are a kind of natural alkaloids. They are widely distributed in the plants and have a variety of physiological activities [12-13]. Daurisoline is a bis-benzylisoquinoline alkaloid extracted from the rhizome of menisperum dauricum, a traditional Chinese medicine [14]. Previous studies showed that daurisoline has potential pharmacological effects on a variety of diseases such as focal ischemia, reperfusion injury, arrhythmia, platelet aggregation, thrombotin A2 inhibition, and so on [13-18]. A few studies found that daurisoline can inhibit the tumor cells proliferation [19-20]. However, the precise mechanism underlying the anticancer effects of daurisoline remains unclear.

Here, quantitative phosphoproteomics was used to explore the potential molecular mechanism of daurisoline in ESCC [21]. Additionally, effect of daurisoline on ESCC tumor growth was evaluated in vivo using the ESCC patient-derived xenograft (PDX) model. Our findings indicated that daurisoline is a MEK1/2 inhibitor and suppresses ESCC growth.

\section{Materials And Methods}

\section{Reagents and antibodies}

Daurisoline was purchased from Shanghai Liding Biotechnology Co., Ltd., (Shanghai, China; purity $\geq 98 \%$, Cat No DR10996) for the in vitro and in vivo analyses. MEK1/2 and ERK1/2 antibodies from Cell Signaling, p-ERK1/2 T185/Y187 antibody from Thermo Fisher Scientific, p-MEK1/2 antibody from Affinity Biosciences Ltd., t-MEK1 and t-MEK2 antibody from Sino Biological, Ki67 antibody from Abcam, $\beta$-tubulin antibody from Hangzhou HuaAn Biotechnology Co., Ltd., and GAPDH antibody from ProteinTech.

\section{Cell culture}

Shantou human embryonic esophageal (SHEE) cells were obtained from Department of Oncology Pathology, Shantou University Medical College; KYSE150 and KYSE450 ESCC cell lines from the Cell Bank of the Chinese Academy of Sciences (Shanghai, China). RMI-1640 medium (Biological Industries, Beit HaEmek, Israel) was used for cells culture. The culture conditions of all cell lines were $37{ }^{\circ} \mathrm{C}$ in a $5 \%$ $\mathrm{CO}_{2}$ atmosphere. 


\section{Cell proliferation assay}

KYSE150 (8000 cells/well), KYSE450 (8000 cells/well), and SHEE (5000 cells/well) cells were seeded in 96-well plates. The media contained different concentrations of daurisoline. All cells were fixed with $4 \%$ paraformaldehyde at $0,24,48,72$ and $96 \mathrm{~h}$. Cells were stained with 4 ', 6-diamino-2-phenylindole (DAPI) and counted by IN Cell Analyzer 6000 (GE Healthcare).

\section{Anchorage-independent growth assay}

The medium containing KYSE150 and KYSE450 cells (8000 cells/well) was added with $0.3 \%$ agar. Then different concentrations of daurisoline were added to the agar. A base layer of $0.6 \%$ agar either contained daurisoline in different concentrations or did not contain any. The cells were cultured at $37{ }^{\circ} \mathrm{C}$ and $5 \%$ $\mathrm{CO}_{2}$ for 10 days, and the number of colonies was counted by IN Cell Analyzer 6000 .

\section{Phosphoproteomics analysis}

After treatment with daurisoline for $24 \mathrm{~h}$, liquid chromatography tandem mass spectrometry (LC-MS/MS) Analysis was performed on the cells protein lysate of KYSE150. Specific experimental methods refer to our previous research [22-23].

\section{Western blotting}

Protein samples (KYSE150 and KYSE450 cells) were obtained from cell lysates (Solarbio, Beijing, China). The protein was electrophoretic by $8 \%$ polyacrylamide gel and then transferred to the PVDF membrane (BIO-RAD). The membranes were blocked with $5 \%$ skimmed milk powder at $25^{\circ} \mathrm{C}$ for $2 \mathrm{~h}$, and the primary antibodies were incubated overnight at $4{ }^{\circ} \mathrm{C}$. They were then incubated with horseradish peroxidase secondary antibody at $25^{\circ} \mathrm{C}$ for $2 \mathrm{~h}$, and the protein bands were detected by eBlot Touch Imager (Shanghai Yibuote Optoelectronic Technology Co., Ltd) using a chemiluminescence reagent.

\section{Molecular docking analysis of daurisoline and MEK1/2 interaction}

First, we used PubChem (https://pubchem.ncbi.nlm.ni h.gov/) to download the 2D structure of daurisoline (PubChem CID: 51106) Next, the crystal structures of MEK1 (3EQH) and MEK2 (1S9I) were downloaded from the Protein Data Bank (PDB) database (https://www.rcsb.org/). All water molecules and small molecule ligands were deleted and hydrogen atoms were added. The docking between daurisoline and MEK1/2 was completed using AutoDock 4.2.6 software (http://autodock.scripps.edu/) under default parameters. Finally, the structure was visualized by PyMOL (The PyMOL Molecular Graphics System, Version 2.3.4).

\section{CNBr-activated sepharose 4B pull down assay}

Active MEK1/2 proteins (250 ng) and KYSE150/450 cells lysates $(500 \mu \mathrm{g})$ were bind to daurisoline-4B sepharose beads. See the previous experiments for details [24]. 


\section{In vitro kinase assay}

The active MEK1 (100 ng) or MEK2 (50 ng) protein was mixed with daurisoline or vehicle (DMSO) at different concentrations in the reaction buffer at $25^{\circ} \mathrm{C}$ for $15 \mathrm{~min}$. Then, the inactive ERK2 protein (250 ng) and ATP $(100 \mu \mathrm{M})$ were added and incubated at $30{ }^{\circ} \mathrm{C}$ for $30 \mathrm{~min}$. Finally, the proteins were analyzed using western blotting.

\section{GSEA analysis}

The GSEA (gene set enrichment analysis) V4.0.3 (http://www.broadinstitute.org/gsea/) was used to analyze the phosphoproteome dataset to observe the difference of protein expression between the daurisoline treatment and control groups. See the references for more details [23].

\section{Spearman's correlation analysis}

The expression level of CDK4/6 gene in esophageal cancer was analyzed by UALCAN website (http://ualcan.path.uab.edu/analysis.html). The two-gene correlation map was realized by ggstatsplot (R package) (https://github.com/Indrajeet Patil/ggstatsplot/), and the multi-gene correlation map was constructed by pheatmap (R package) (https://cran.r-project.org/web/packages/pheatmap/). $P<0.05$ denoted statistical significance.

\section{Establishment of the ESCC PDX model}

The tumor sample from an ESCC patient was EG20 (ESCC, $\mathrm{T}_{2} \mathrm{~N}_{0} \mathrm{M}_{0} \mathrm{ll}$, moderately differentiated, obtained from Linzhou Cancer Hospital, Henan Province, China). The daurisoline treatment group was given 20 and $40 \mathrm{mg} / \mathrm{kg}$, respectively. More experimental details are borrowed from our previous method [25].

\section{Immunohistochemical staining}

Immunochemical staining was used to detect the molecular changes in EG20 tumors. The indexes were Ki67, p-MEK1/2, and p-ERK1/2. See our previous instructions for the complete experiment [25].

\section{Statistical analyses}

SPSS 24.0 software (IBM, USA) was used for data analysis in this experiment, and the results were expressed as Mean \pm SD. Student T-test, one-way ANOVA or Kruskal-Wallis H-test was used according to different conditions of the data. The experiment was repeated three times, and $p<0.05$ indicated statistically significant difference.

\section{Results}

\section{Daurisoline inhibits ESCC cell proliferation in vitro}


By drug screening, $40 \mu \mathrm{M}$ daurisoline was found to have obvious cytotoxicity to KYSE450 cells (Fig. 1a). Daurisoline is a bis-benzylisoquinoline alkaloid (Fig. 1b). The IC ${ }_{50}$ of KYSE150 and KYSE450 cells were 20.733 and $13.560 \mu \mathrm{M}$ at $24 \mathrm{~h}$, and 11.0149 and $9.936 \mu \mathrm{M}$ at $48 \mathrm{~h}$, respectively. Under daurisoline treatment for $24 \mathrm{~h}$ and $48 \mathrm{~h}, \mathrm{IC}_{50}$ of immortalized esophageal epithelial cells (SHEE cells) was $41.102 \mu \mathrm{M}$ and $11.406 \mu \mathrm{M}$, respectively. With the increase in daurisoline concentration, the survival rate of cells decreased gradually (Fig. 1c). In the cell proliferation assay, we used the following drug concentrations: 0 , $2.5,5,10$, and $20 \mu \mathrm{M}$. Daurisoline inhibited the proliferation of KYSE150 and KYSE450 cells in a dosedependent manner. The proliferation inhibition rates of KYSE150, KYSE450, and SHEE cells were 44.51\%, $68.83 \%$, and $25.50 \%$, respectively, after treatment with $20 \mu \mathrm{M}$ daurisoline for $96 \mathrm{~h}$ (Fig. $1 \mathrm{~d}$ ). These results indicated that daurisoline was less toxic to SHEE cells compared with ESCC cells. To verify this, we further performed the anchorage-independent cell growth assay using the same drug concentration. Daurisoline inhibited the clonal size and number of clones formed for KYSE150 and KYSE450 cells. At 20 $\mu \mathrm{M}$, the clonal formation rates of KYSE150 and KYSE450 cells were $30.25 \%$ and $42.36 \%$, respectively, compared with that of the control cells (Fig. 1e). These results indicated that daurisoline inhibited ESCC cell proliferation in vitro.

\section{Daurisoline significantly downregulates the phosphorylation of ERK1/2 in ESCC cells}

We used quantitative phosphoproteomics to investigate the molecular mechanism underlying daurisoline inhibiting ESCC cell proliferation. We treated KYSE150 cells with $20 \mu \mathrm{M}$ daurisoline for $24 \mathrm{~h}$. (Fig. 2a). The first-order mass error of most spectrograms was within $10 \mathrm{ppm}$, which conformed to the high precision characteristic of orbital well MS (Fig. S1a). Most peptides were 7-20 amino acids in length. The distribution of the peptide lengths identified by MS conformed to the quality control requirements (Fig. S1b). The number of secondary spectra obtained was 383,273 . After screening, the number of available effective spectrograms was 94,520 . We identified 12,965 phosphorylation sites on 3130 proteins, of which 7415 sites on 2500 proteins contained quantitative data. To ensure a high credibility of the results, $p>0.75$ was used to filter the identification data. Consequently, we identified 8549 sites on 2861 proteins, of which 6601 sites on 2408 proteins included quantitative data (Fig. S1c). The protein quantification group was normalized to remove the influence of protein expression on the modified signal. The screening criteria for different sites were as follows: 1.5 times the change threshold and two-sample twotailed $t$-test $(p<0.05)$. Based on these data and standards, we found that the phosphorylation of 176 sites was enhanced and that of 340 sites was reduced in the daurisoline treated cells (Fig. S1d).

A total of 340 down regulated phosphorylation sites were enriched in the Kyoto Encyclopedia of genes and genomes (KEGG) pathway. KEGG pathway was selected according to Fisher's exact test $(p<0.05)$. Ultimately, nine KEGG pathway targets were screened: viral carcinogenesis, spliceosome, RNA transport, adipocytokine signaling pathway, herpes simplex virus infection, synaptic vesicle cycle, Salmonella infection, chronic myeloid leukemia and Th17 cell differentiation (Fig. 2b). The heat map shows the levels of the phosphorylation sites in the viral carcinogenesis pathway, and most of the phosphorylation sites are downregulated (Fig. 2c). In six KEGG pathways, multiple proteins appeared repeatedly in the different pathways. Therefore, Wayne diagrams are made based on the six KEGG pathways to find the key proteins 
in these pathways (Fig. 2d). We found that NFKB1 was enriched in six pathways, ERK2 in four pathways, and STAT3 in three pathways. The original mass spectra of p-ERK2 T185/Y187 are shown in Supplement Figure (Fig. S1e). In the volcanic map, according to the - $\log _{10}(P$-value), phosphorylation of the four phosphorylation sites is ranked from top to bottom as p-ERK2 Y187 (3.0177), p-ERK2 T185 (2.4976), pSTAT3 S727 (1.7433), and p-NFKB1 S907 (1.5686) (Fig. 2e). In addition, the protein expression ratios of the above four phosphorylation sites are $0.504,0.458,0.566$, and 0.503 , respectively (Fig. 2f). In conclusion, we believe that p-ERK2 T185/Y187 are important phosphorylation sites in the above nine KEGG pathways. In addition, although ERK1 was not enriched in the KEGG pathway, the phosphorylation of ERK1 was also downregulated in the phosphoproteomics data. The phosphorylation level of ERK1/2 in KYSE150 and KYSE450 cells was significantly downregulated after daurisoline $(20 \mu \mathrm{M})$ treatment for 24 h (Fig. 2g). These results indicated that daurisoline inhibited ERK1/2 phosphorylation in ESCC cells.

\section{Daurisoline directly binds with MEK1 /2}

We used IGPS1.0 software to predict upstream kinases for specific phosphorylation sites in the above nine KEGG pathways. The prediction results showed that the upstream kinase proteins of p-ERK2 T185 and p-ERK2 Y187 were MEK1-7 (MAP2K1-7), and ALK and LTK, respectively (Table. S1). In order to verify the accuracy of the upstream kinase prediction, we performed the CNBr-activated Sepharose 4B pull down assay (Fig. S2). The protein binding to the drug was detected by Mass spectrum. The results of mass spectrometry showed that the protein binding to daurisoline were MEK1, MEK2, MEK3, and MEK7 with Unused ProtScores of 38.69, 17.28, 15.76, and 4.83, respectively (Table S2). Previous studies on MAPK signaling pathway showed that the sole substrate of MEK $1 / 2$ protein kinase is ERK $1 / 2$, the substrate of MEK3 is p38MAPK, and the substrate of MEK7 is JNK [26]. Therefore, based on the Unused ProtScores, daurisoline was likely to affect the biological function of MEK1/2 and downregulate ERK1/2 phosphorylation.

Autodock 4.0 software was used for molecular docking in order to explore the specific binding sites of daurisoline with MEK1/2 proteins. Daurisoline could bind to MEK1 (binding energy: $-11.06 \mathrm{kcal} / \mathrm{mol}$ ). The binding sites were Asn78 and Lys97 (Fig. 3a). Daurisoline could also bind to MEK2 (binding energy: -8.50 $\mathrm{kcal} / \mathrm{mol}$ ). The binding sites were Asp194 and Asp212 (Fig. 3b). Pull down assays displayed that daurisoline could directly bind to MEK1/2 (Fig. 3c, d). In addition, daurisoline also binds directly to MEK1 and MEK2 in KYSE150 and KYSE450 cells (Fig. 3e, f). To verify the accuracy of the molecular simulation results, we substituted the above-mentioned amino acids of MEK1 and MEK2 proteins with alanine (Ala, A). The results showed that after Asn78 substitution on MEK1 protein, the binding efficiency of the protein with daurisoline was significantly reduced (Fig. $3 \mathrm{~g}$ ). Furthermore, the binding efficiency of MEK2 protein with daurisoline was significantly reduced after Asp194 substitution (Fig. 3h).

\section{Daurisoline inhibits the MEK1/2-ERK1/2 signaling pathway in ESCC}

We performed an in vitro kinase assay. The phosphorylation efficiency of active MEK1 and MEK2 against inactive ERK2 was in turn reduced at 2.5, 5, 10, and $20 \mu \mathrm{M}$ daurisoline (Fig. 4a, b). These results indicated 
that daurisoline could inhibit ERK2 phosphorylation by inhibiting MEK1/2 activity. Moreover, Coomassie blue staining showed the location and expression of MEK1/2 and ERK2 proteins.

We used gene set enrichment analysis (GSEA) to enrich the KEGG pathway associated with altered expression of all proteins identified in the phosphoproteomics analysis. We found that ERK $1 / 2$ was enriched and significantly downregulated in the Erbb signaling pathway (Fig. 4c) and prostate cancer pathway (Fig. 4d). In addition, MEK2 (MAP2K2) was also enriched in the aforementioned two pathways. Western blotting showed that daurisoline inhibited ERK1/2 phosphorylation in KYSE150 and KYSE450 cells. However, daurisoline had no significant effect on MEK1/2 phosphorylation (Fig. 4c, d). The above results indicate that daurisoline inhibits the MEK1/2-ERK1/2 signaling pathway in ESCC cells.

\section{MEK1/2 were significantly overexpressed in ESCC}

MEK1/2 have been reported to be highly expressed in ESCC [27]. The Cancer Genome Atlas (TCGA) database revealed that MEK1 (Fig. 5a) and MEK2 (Fig. 5b) were both significantly overexpressed in a variety of cancers, including ESCA. In addition, MEK1 (Fig. 5c) and MEK2 (Fig. 5d) are also overexpressed in EAC and ESCC. We used ESCC data from the TCGA database to conduct a multi-gene correlation analysis. We observed a significant correlation between MEK1 and ERK2 in ESCC (Fig. 5f, g).

\section{Daurisoline inhibits ESCC tumor growth in vivo}

To investigate the anticancer effect of daurisoline in vivo, we established the PDX model of ESCC (Fig. 6a). There was no significant difference in the body weight between mice treated with daurisoline and control, indicating that daurisoline had no toxic or side effects on mice (Fig. 6b). The final tumor volume was measured at the end of the treatment (Fig. 6c). The tumor growth rate of daurisoline treated group was significantly lower than that of control group (Fig. $6 \mathrm{~d}, \mathrm{~g}$ ). The tumor weight also decreased with the increase of the dose of daurisoline (Fig. 6e). We calculated the tumor weight inhibition rate of daurisoline treated group. The growth inhibition rates in the 20 and $40 \mathrm{mg} / \mathrm{kg}$ daurisoline treatment groups were $52.77 \%$ and $84.74 \%$, respectively (Fig. 6 f). Immunohistochemical staining showed that the Ki67 and ERK1/2 phosphorylation decreased after daurisoline treatment. The MEK1/2 phosphorylation did not change significantly (Fig. 6h). In conclusion, daurisoline inhibited ESCC tumor proliferation and ERK1/2 phosphorylation in tumor cells in vivo.

\section{Discussion}

It is of great clinical significance to find drugs that can prevent the occurrence and recurrence of ESCC [6]. Traditional Chinese medicine has long been used in clinical settings. Compounds extracted from traditional Chinese medicine represent a promising resource for anticancer drug development [28]. By screening natural compounds, we found that daurisoline exhibits anticancer effects on ESCC. We found that daurisoline inhibited the proliferation of ESCC cells. In addition, daurisoline was less toxic to SHEE cells (Fig. 1c, d). These results indicate that at the cellular level, the anticancer effect of daurisoline is more beneficial than harmful. Furthermore, PDX model can retain the histological, molecular, and genetic 
characteristics of the original tumor; thus, it is superior to the traditional cell lines and their derivatives in vitro [29-30]. In this study, we observed that daurisoline displayed a significant anticancer effect in vivo (Fig. 6e, f). The anticancer effect of daurisoline in ESCC PDX model was also significantly better than that of CDX model of lung cancer [19].

Phosphorylation regulates many aspects of protein function. It is a dynamic post-translational modification, which alters protein structure, localization, interaction, and stability [31]. Although a few literatures have reported the anticancer effects of daurisoline in vitro and in vivo, the mechanistic target of daurisoline remains undetermined [18-20]. Therefore, in this study, we explored the mechanism underlying daurisoline inhibiting ESCC cell proliferation through phosphoproteomics. The results of mass spectrometry showed that daurisoline inhibited ERK1/2 phosphorylation in ESCC cells (Fig. 2g). We finally identified the molecular targets of daurisoline as MEK1/2. Moreover, In vitro kinase assay showed that daurisoline inhibited ERK2 phosphorylation by inhibiting MEK1/2 activity (Fig. 4a, b). In addition, we performed a polygenetic correlation analysis on ESCC clinical samples from the TCGA database, and found that MEK1 and ERK2 were significantly correlated (Fig. 5e, f).

MEK1/2 kinases can specifically phosphorylate and activate ERK1/2 [32]. Abnormal regulation of the MEK1/2-ERK1/2 pathway will inevitably lead to cell cancerization [33]. As a key nodal protein in this signaling pathway, MEK1/2 plays an important role in tumorigenesis [34]. MEK1/2, as the target of tumor therapy, is highly selective and unique [35-36]. MEK1 and MEK2 share $80 \%$ of the amino acid composition in the kinase domain and are highly conserved evolutionarily [37]. The interaction between natural compounds and target proteins can be simulated by molecular docking technology [38]. We observed that daurisoline bound to MEK1 at Asn78 and Lys97 sites, and MEK2 at Asp194 and Asp212 sites (Fig. 3a, b). It was found that Asn78 residue of MEK1 and Asp194 residue of MEK2 were important binding sites for daurisoline (Fig. 3g, h).

Therefore, our findings showed that daurisoline inhibited the growth of ESCC tumor by inhibiting the MEK1/2-ERK1/2 signaling pathway (Fig. 6i). Daurisoline may prevent ESCC in high-risk individuals and reduce the postoperative recurrence rate of ESCC.

\section{Conclusions}

In this study, we demonstrated that daurisoline is a MEK1/2 inhibitor, which led to a decrease in ERK1/2 phosphorylation. This in turn leads to inhibition of ESCC cells proliferation and tumor growth in the ESCC PDX model.

\section{Abbreviations}

ESCA: Esophageal cancer; ESCC: Esophageal squamous cell carcinoma; EAC: Esophageal adenocarcinoma; SHEE: Shantou human embryonic esophageal; PDX: patient-derived xenograft; CDX: cell-derived xenograft; SCID: severe combined immunodeficiency; LC-MS/MS: liquid chromatography 
tandem mass spectrometry; GSEA: gene set enrichment analysis; TCGA: The Cancer Genome Atlas;

KEGG: Kyoto Encyclopedia of genes and genomes.

\section{Declarations}

\section{Acknowledgments}

Thanks to Bo Li, Xining Liu, Yubing Zhou, Zhuo Bao, Yaxing Wei, Xiangyu Wu and Ang Li from the China US (Henan) Hormel cancer institute for their help.

\section{Authors' contributions}

Donghao Wang participated in the whole experiment and wrote the original manuscript. Ning Yang and Xiaofan Zhang conducted cell experiments. Mingzhu Li performed site-directed mutation experiments on amino acids. Lili Zhao carried out the immunohistochemical staining experiment. Qiang Yuan and Yin Yu extracted the purified protein. Jing Lu and Jimin Zhao analyzed the data. Yanan Jiang and Xin Li revised the paper. Zigang Dong and Kangdong Liu directed the design of the project. All authors have read and approved the final manuscript.

\section{Funding}

This research was supported by the National Natural Science Foundations of China (No. 81872335; No. 32000650), National Natural Science Youth Foundation (No. 81902486), National Science \& Technology Major Project Key New Drug Creation and Manufacturing Program, China (No. 2018ZX09711002), Natural Science Foundation of Henan (No. 161100510300), Science and Technology Project of Henan Province (No. 212102310187).

\section{Availability of data and materials}

All the data and materials that support conclusions are included in the main paper.

\section{Ethics approval and consent to participate}

The research proposals of ESCC PDX mice model are approved by the research Ethics Committee of Zhengzhou University.

\section{Consent for publication}

All the authors have agreed to publish this article.

\section{Competing interests}

The authors declare no conflict of interest.

\section{Author details}


${ }^{1}$ Pathophysiology Department, The School of Basic Medical Sciences, Zhengzhou University, Zhengzhou, Henan, 450001, China. ${ }^{2}$ China-US (Henan) Hormel Cancer Institute, Zhengzhou, Henan, 450008, China.

${ }^{3}$ Collaborative Innovation Center of Henan Province for Cancer Chemoprevention, Zhengzhou University, Zhengzhou, Henan, China. ${ }^{4}$ Cancer chemoprevention international collaboration laboratory, Zhengzhou, Henan, China. ${ }^{5}$ State Key Laboratory of Esophageal Cancer Prevention and Treatment, Zhengzhou, Henan, China.

\section{References}

1. Sung H, Ferlay J, Siegel RL, Laversanne M, Soerjomataram I, Jemal A, et al. Global cancer statistics 2020: GLOBOCAN estimates of incidence and mortality worldwide for 36 cancers in 185 countries. CA Cancer J Clin. 2021.

2. Smyth EC, Lagergren J, Fitzgerald RC, Lordick F, Shah MA, Lagergren P, et al. Oesophageal cancer. Nat Rev Dis Primers. 2017;3:17048.

3. Abnet CC, Arnold M, Wei WQ. Epidemiology of Esophageal Squamous Cell Carcinoma. Gastroenterology. 2018;154(2):360-373.

4. di Pietro M, Canto MI, Fitzgerald RC. Endoscopic Management of Early Adenocarcinoma and Squamous Cell Carcinoma of the Esophagus: Screening, Diagnosis, and Therapy. Gastroenterology. 2018;154(2):421-436.

5. Codipilly DC, Qin Y, Dawsey SM, Kisiel J, Topazian M, Ahlquist D, et al. Screening for esophageal squamous cell carcinoma: recent advances. Gastrointest Endosc. 2018;88(3):413-426.

6. Talukdar FR, di Pietro M, Secrier M, Moehler M, Goepfert K, Lima S, et al. Molecular landscape of esophageal cancer: implications for early detection and personalized therapy. Ann N Y Acad Sci. 2018;1434(1):342-359.

7. Wu PK, Becker A, Park JI. Growth Inhibitory Signaling of the Raf/MEK/ERK Pathway. Int J Mol Sci. 2020;21(15).

8. Burotto M, Chiou VL, Lee JM, Kohn EC. The MAPK pathway across different malignancies: a new perspective. Cancer-Am Cancer Soc. 2014;120(22):3446-3456.

9. Cheng Y, Tian H. Current Development Status of MEK Inhibitors. Molecules. 2017;22(10).

10. Faghfuri E, Nikfar S, Niaz K, Faramarzi MA, Abdollahi M. Mitogen-activated protein kinase (MEK) inhibitors to treat melanoma alone or in combination with other kinase inhibitors. Expert Opin Drug Metab Toxicol. 2018;14(3):317-330.

11. Kun E, Tsang Y, Ng CW, Gershenson DM, Wong KK. MEK inhibitor resistance mechanisms and recent developments in combination trials. Cancer Treat Rev. 2021;92:102137.

12. Weber C, Opatz T. Bisbenzylisoquinoline Alkaloids. Alkaloids Chem Biol. 2019;81:1-114.

13. Qian JQ. Cardiovascular pharmacological effects of bisbenzylisoquinoline alkaloid derivatives. Acta Pharmacol Sin. 2002;23(12):1086-1092. 
14. Liu Q, Mao X, Zeng F, Jin S, Yang X. Effect of daurisoline on HERG channel electrophysiological function and protein expression. J Nat Prod. 2012;75(9):1539-1545.

15. Zhao B, Chen Y, Sun X, Zhou M, Ding J, Zhan J, et al. Phenolic alkaloids from Menispermum dauricum rhizome protect against brain ischemia injury via regulation of GLT-1, EAAC1 and ROS generation. Molecules (Basel, Switzerland). 2012;17(3):2725-2737.

16. Jiang Q, Peterson AM, Chu Y, Yao X, Zha X, Chu X. Histidine Residues Are Responsible for Bidirectional Effects of Zinc on Acid-Sensing Ion Channel 1a/3 Heteromeric Channels. Biomolecules. 2020;10(9).

17. Wang $P$, Luo $R$, Zhang $M$, Wang $Y$, Song $T$, Tao $T$, et al. A cross-talk between epithelium and endothelium mediates human alveolar-capillary injury during SARS-CoV-2 infection. Cell Death Dis. 2020;11(12):1042

18. Osmakov DI, Koshelev SG, Lyukmanova EN, Shulepko MA, Andreev YA, Illes P, et al. Multiple Modulation of Acid-Sensing Ion Channel 1a by the Alkaloid Daurisoline. Biomolecules. 2019;9(8).

19. Huang XH, Yan X, Zhang QH, Hong P, Zhang WX, Liu YP, et al. Direct targeting of HSP90 with daurisoline destabilizes beta-catenin to suppress lung cancer tumorigenesis. Cancer Lett. 2020;489:66-78.

20. Xue L, Liu P. Daurisoline inhibits hepatocellular carcinoma progression by restraining autophagy and promoting cispaltin-induced cell death. Biochem Biophys Res Commun. 2021;534:1083-1090.

21. Noujaim J, Payne LS, Judson I, Jones RL, Huang PH. Phosphoproteomics in translational research: a sarcoma perspective. Ann Oncol. 2016;27(5):787-794.

22. Yuan Q, Dong CD, Ge Y, Chen X, Li Z, Li X, et al. Proteome and phosphoproteome reveal mechanisms of action of atorvastatin against esophageal squamous cell carcinoma. Aging (Albany NY). 2019;11(21):9530-9543.

23. Li Z, Li X, He X, Jia X, Zhang X, Lu B, et al. Proteomics Reveal the Inhibitory Mechanism of Levodopa Against Esophageal Squamous Cell Carcinoma. Front Pharmacol. 2020;11:568459.

24. Jia X, Huang C, Hu Y, Wu Q, Liu F, Nie W, et al. Cirsiliol targets tyrosine kinase 2 to inhibit esophageal squamous cell carcinoma growth in vitro and in vivo. Journal of experimental \& clinical cancer research: CR. 2021;40(1):105.

25. Zhu Lili CXZY. Dihydroartemisinin Inhibits the Proliferation of Esophageal Squamous Cell Carcinoma Partially by Targeting AKT1 and p70S6K. Front Pharmacol. 2020;11:1850.

26. Cargnello M, Roux PP. Activation and function of the MAPKs and their substrates, the MAPKactivated protein kinases. Microbiol Mol Biol Rev. 2011;75(1):50-83.

27. Nan P, Wang T, Li C, Li H, Wang J, Zhang J, et al. MTA1 promotes tumorigenesis and development of esophageal squamous cell carcinoma via activating the MEK/ERK/p90RSK signaling pathway. Carcinogenesis. 2020;41(9):1263-1272.

28. Wang K, Chen Q, Shao Y, Yin S, Liu C, Liu Y, et al. Anticancer activities of TCM and their active components against tumor metastasis. Biomed Pharmacother. 2021;133:111044. 
29. Lai Y, Wei X, Lin S, Qin L, Cheng L, Li P. Current status and perspectives of patient-derived xenograft models in cancer research. J Hematol Oncol. 2017;10(1):106.

30. Yoshida GJ. Applications of patient-derived tumor xenograft models and tumor organoids. $J$ Hematol Oncol. 2020;13(1):4.

31. Harsha HC, Pandey A. Phosphoproteomics in cancer. Mol Oncol. 2010;4(6):482-495.

32. Yuan J, Dong X, Yap J, Hu J. The MAPK and AMPK signalings: interplay and implication in targeted cancer therapy. J Hematol Oncol. 2020;13(1):113.

33. Khan AQ, Kuttikrishnan S, Siveen KS, Prabhu KS, Shanmugakonar M, Al-Naemi HA, et al. RASmediated oncogenic signaling pathways in human malignancies. Semin Cancer Biol. 2019;54:1-13.

34. Burotto M, Chiou VL, Lee JM, Kohn EC. The MAPK pathway across different malignancies: a new perspective. Cancer-Am Cancer Soc. 2014;120(22):3446-3456.

35. Kim C, Giaccone G. MEK inhibitors under development for treatment of non-small-cell lung cancer. Expert Opin Investig Drugs. 2018;27(1):17-30.

36. Barbosa R, Acevedo LA, Marmorstein R. The MEK/ERK Network as a Therapeutic Target in Human Cancer. Mol Cancer Res. 2021;19(3):361-374.

37. Catalanotti F, Reyes G, Jesenberger V, Galabova-Kovacs G, de Matos SR, Carugo O, et al. A Mek1Mek2 heterodimer determines the strength and duration of the Erk signal. Nat Struct Mol Biol. 2009;16(3):294-303.

38. Chen Y, Shoichet BK. Molecular docking and ligand specificity in fragment-based inhibitor discovery. Nat Chem Biol. 2009;5(5):358-364.

\section{Figures}


Fig. 1

a

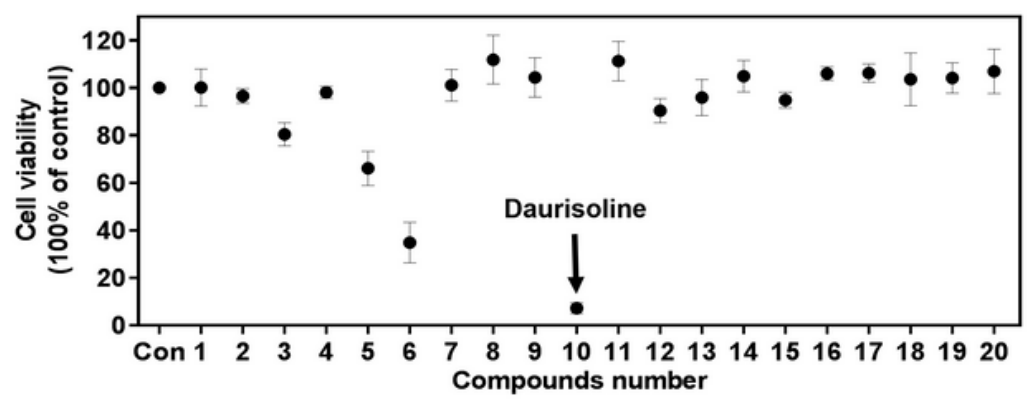

KYSE 150

C

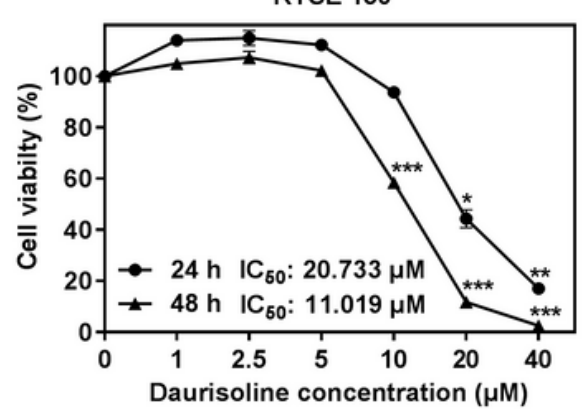

d

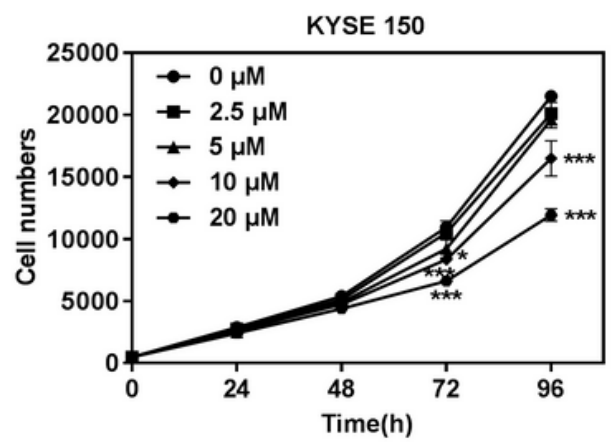

KYSE 450

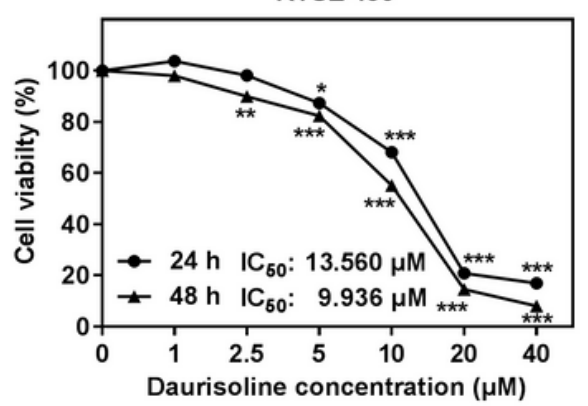

KYSE 450

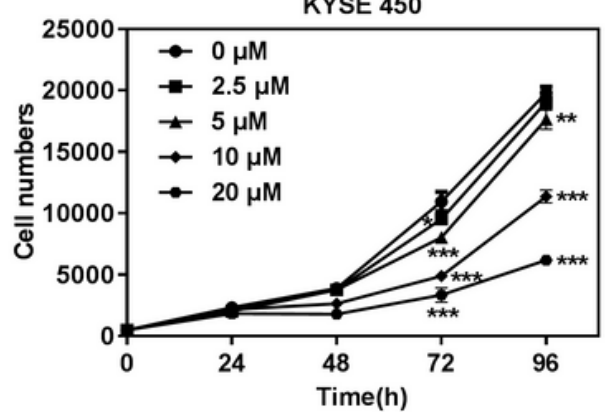

b

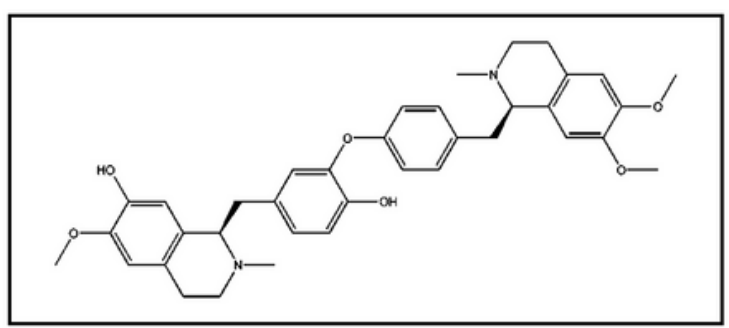

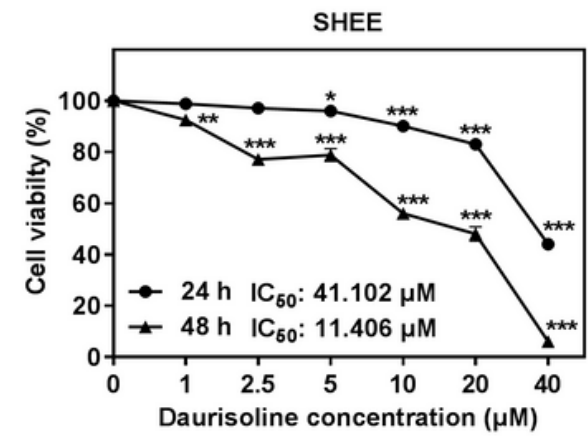

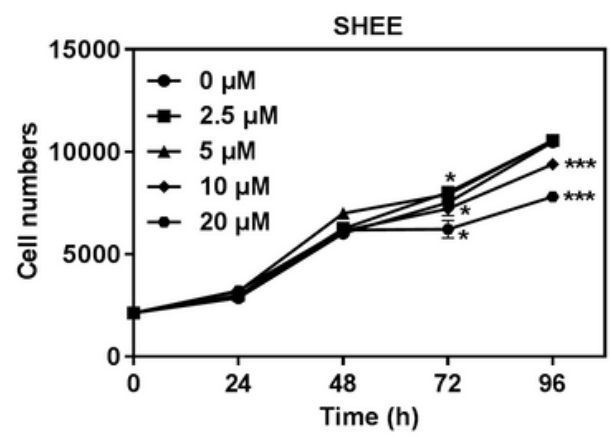

e

Daurisoline concentration ( $\mu \mathrm{M})$
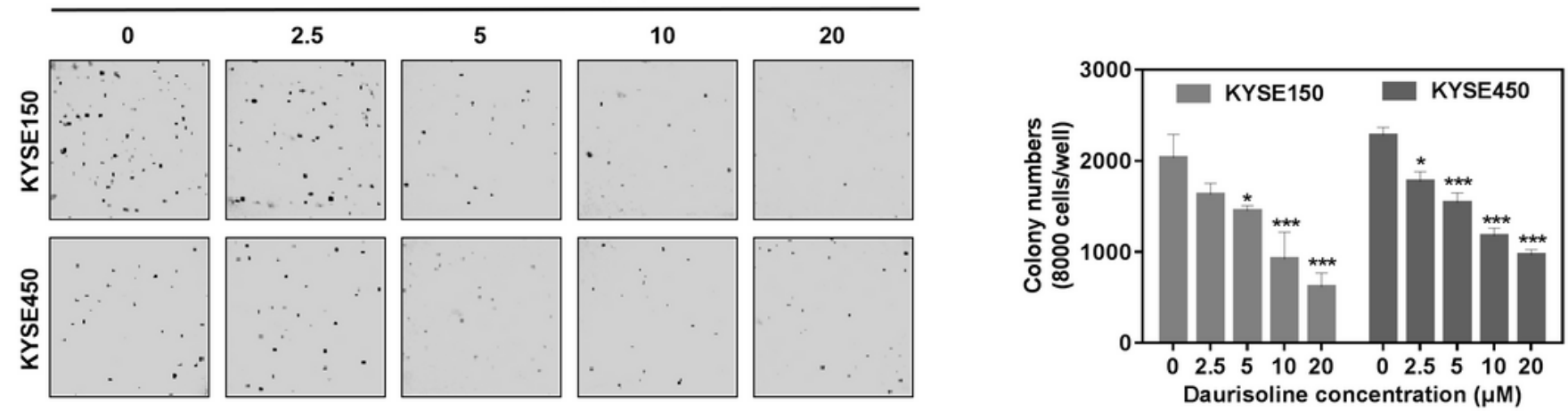

Figure 1

Daurisoline inhibits ECSS cells proliferation in vitro. a Survival rate of KYSE450 cells after $48 \mathrm{~h}$ treatment with different drugs. Number 10 (arrow) is daurisoline. b Chemical structural formula for daurisoline. $c$ The toxicity assay of KYSE150, KYSE450 and SHEE cells. Cells were treated with daurisoline $(0,1,2.5,5$, 10, 20 and $40 \mu \mathrm{M}$ ) for 24 and $48 \mathrm{~h}$. d The cell proliferation assay of KYSE150, KYSE450 and SHEE cells. Cells were treated with daurisoline $(0,2.5,5,10$ and $20 \mu \mathrm{M})$ for $24,48,72$ and $96 \mathrm{~h}$. e Effect of daurisoline 
on anchorage-independent growth of KYSE150 and KYSE450 cells. All data are shown as a triplicate mean \pm SD of values from 3 independent trials. ( $\mathrm{P}<0.05, * * P<0.01$, ***P $<0.001$ )

Fig. 2

a

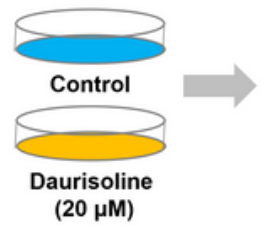

b

d

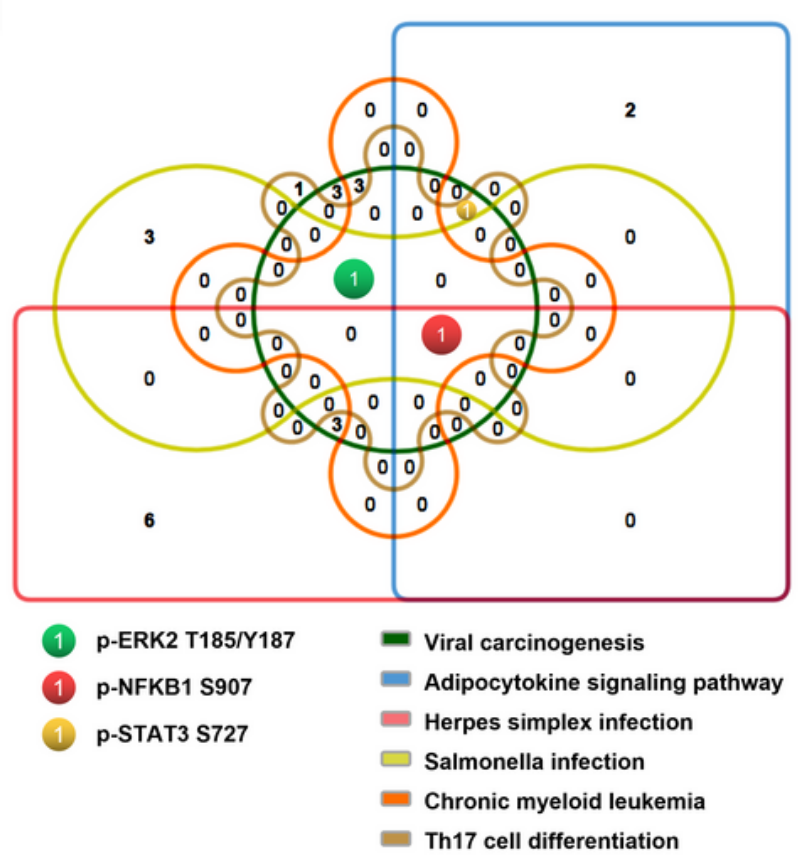

f

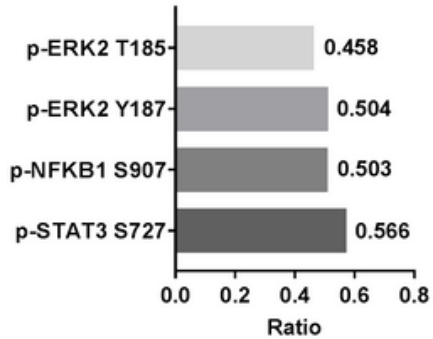

g

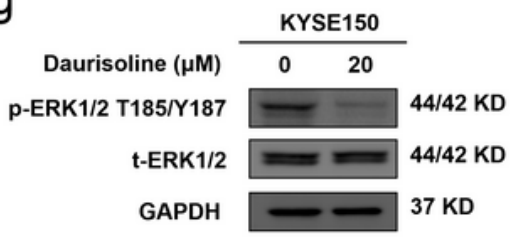

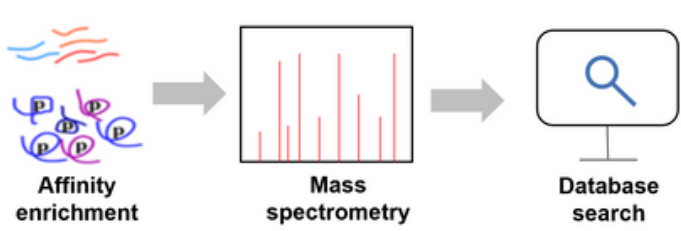

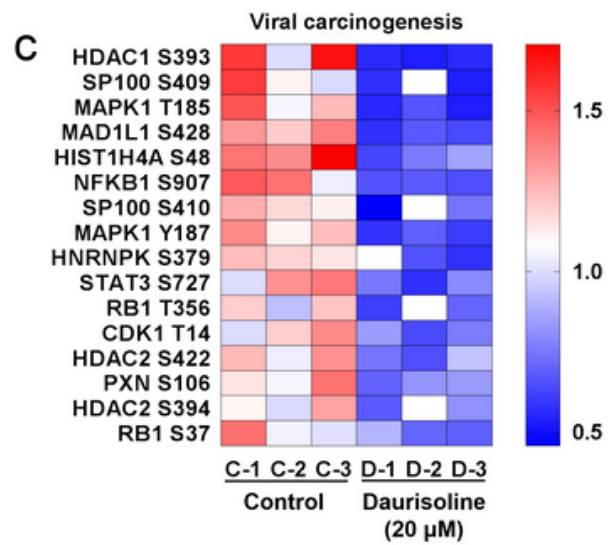

e
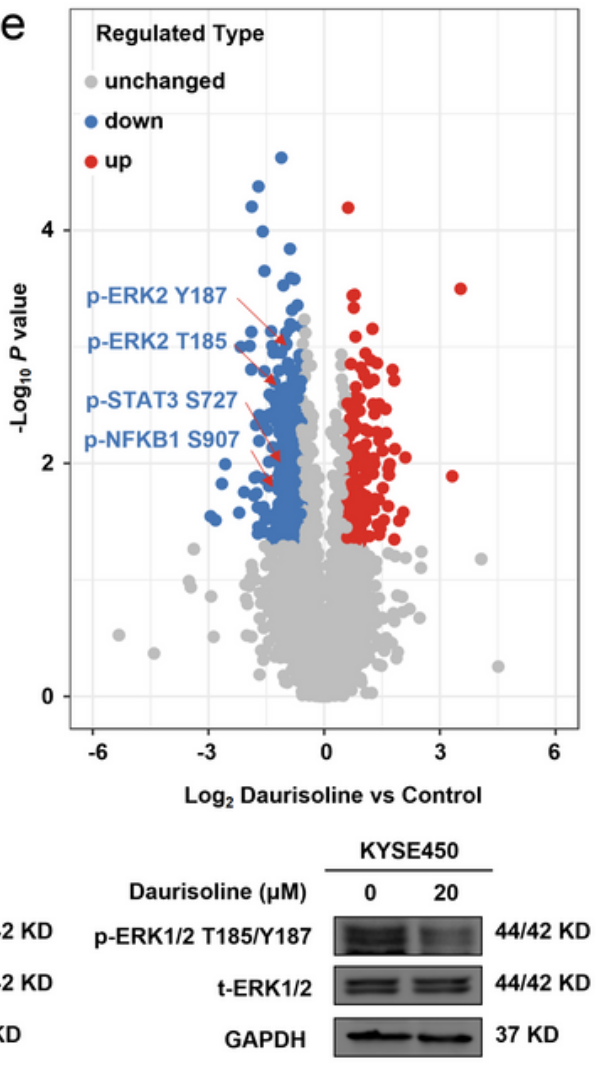

\section{Figure 2}

Daurisoline significantly downregulates the phosphorylation of ERK1/2 in ESCC cells. a The flow chart of the phosphoproteomics analysis of KYSE150 cells treated by Daurisoline. b The enrichment analysis of KEGG pathway at downregulated protein phosphorylation sites (Fisher's exact test $\mathrm{P}$ value $<0.05$ ). $\mathrm{c} \mathrm{Heat}$ 
map of downregulated phosphorylation sites in the viral carcinogenesis pathway. $d$ Wayne Diagram of six down-regulated KEGG pathways. e Volcano plot of differentially expressed modification sites. The red dots indicate the sites with significantly up-regulated expression, and the blue dots indicate the sites with significantly down-regulated expression. $f$ Ratio values of 4 protein phosphorylation sites. $g$ Western blot of $\mathrm{p}$-ERK1/2 T185/Y187.

Fig. 3
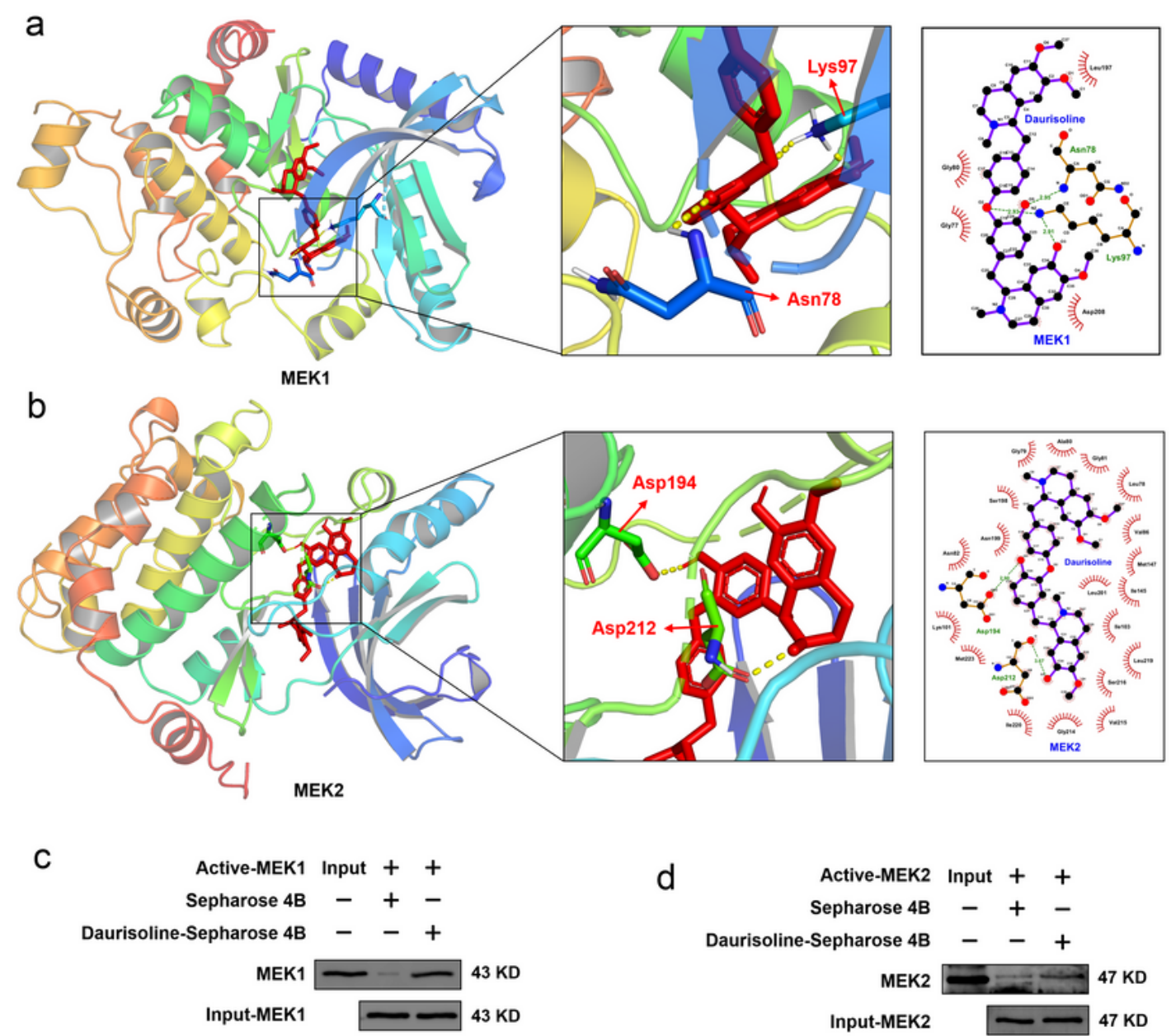
e KYSE 150 cell lysate Input ++ Sepharose $4 \mathrm{~B}-+$ Daurisoline-Sepharose 4B $-\quad-+$

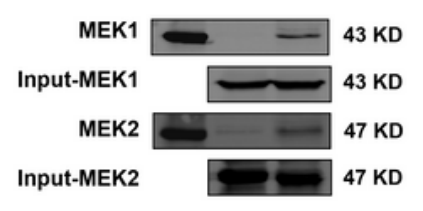

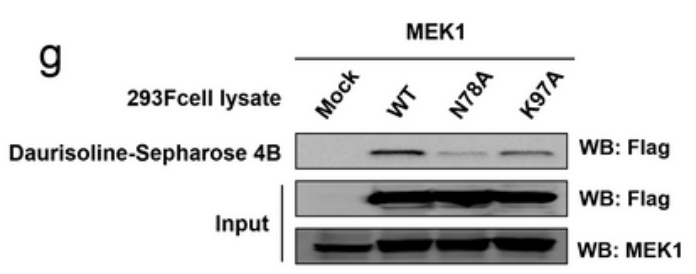
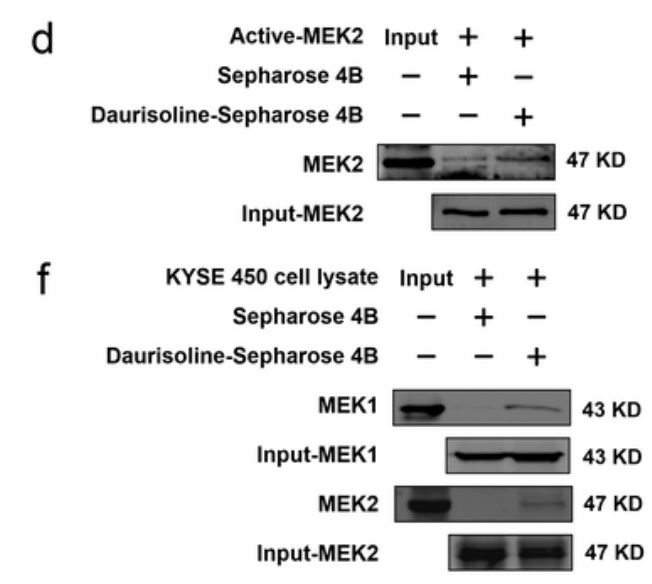

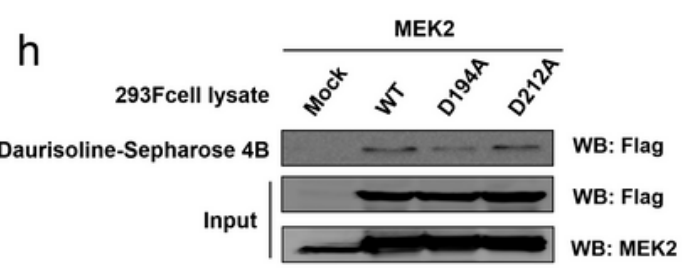

Figure 3 
Daurisoline directly binds with MEK1 and MEK2. a The 3D binding model of daurisoline and MEK1 (left panel). The red arrows show the amino acids forming hydrogen bonds with daurisoline, and the yellow dotted lines show hydrogen bonds. The 2D binding model of daurisoline and MEK1 (right panel). The green dotted line represents the hydrogen bond, and the green number is the length of the hydrogen bond. b The 3D binding model of daurisoline and MEK2 (left panel). The 2D binding model of daurisoline and MEK2 (right panel). c,d The pull down assays of Active-MEK1/2 and daurisoline. e,f The pull down assays of KYSE150/450 cell lysate and daurisoline. g,h The pull down assays of daurisoline and amino acid site mutation of MEK1/2 protein.

\section{Fig. 4}

a

$\begin{array}{rccccccc}\text { Daurisoline } & - & - & \text { DMSO } & 2.5 & 5 & 10 & 20 \\ \text { Active-MEK1 } & + & - & + & + & + & + & + \\ \text { inactive ERK2 } & - & - & + & + & + & + & + \\ \text { ATP } & + & + & + & + & + & + & +\end{array}$

p-ERK2 T185/Y187 $\square-\cdots$
t-ERK2

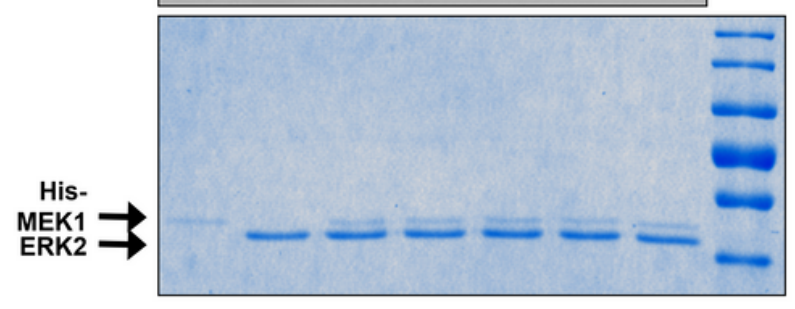

b

\begin{tabular}{rccccccc}
\multicolumn{10}{c}{ Daurisoline } & - & - & DMSO & 2.5 & 5 & 10 & 20 \\
\cline { 2 - 8 } Active-MEK2 & + & - & + & + & + & + & + \\
inactive ERK2 & - & - & + & + & + & + & + \\
ATP & + & + & + & + & + & + & +
\end{tabular}

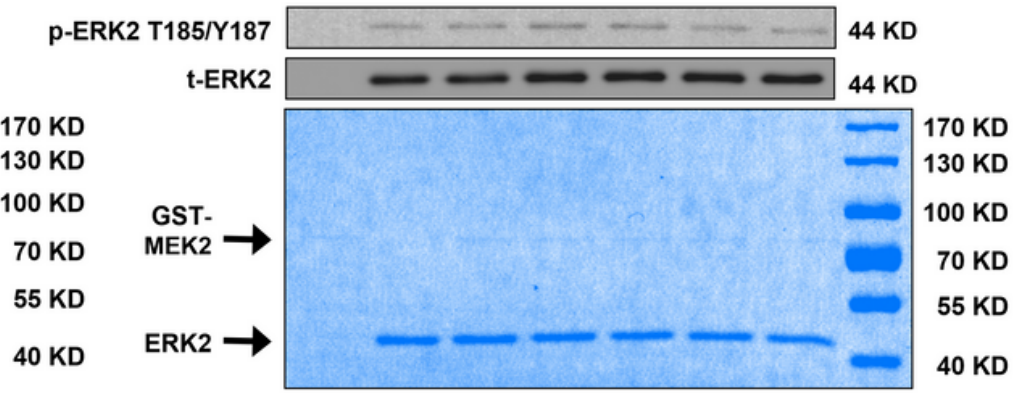

C

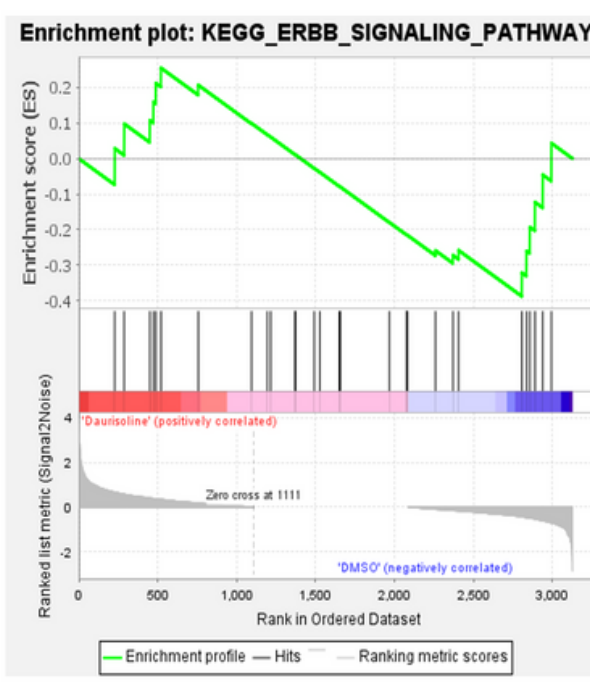

e

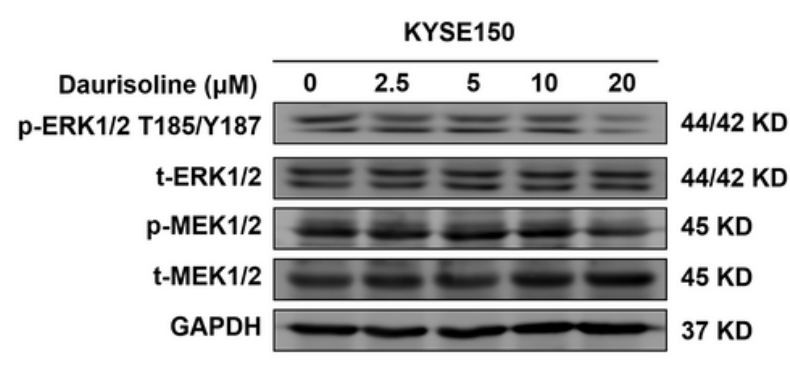

d
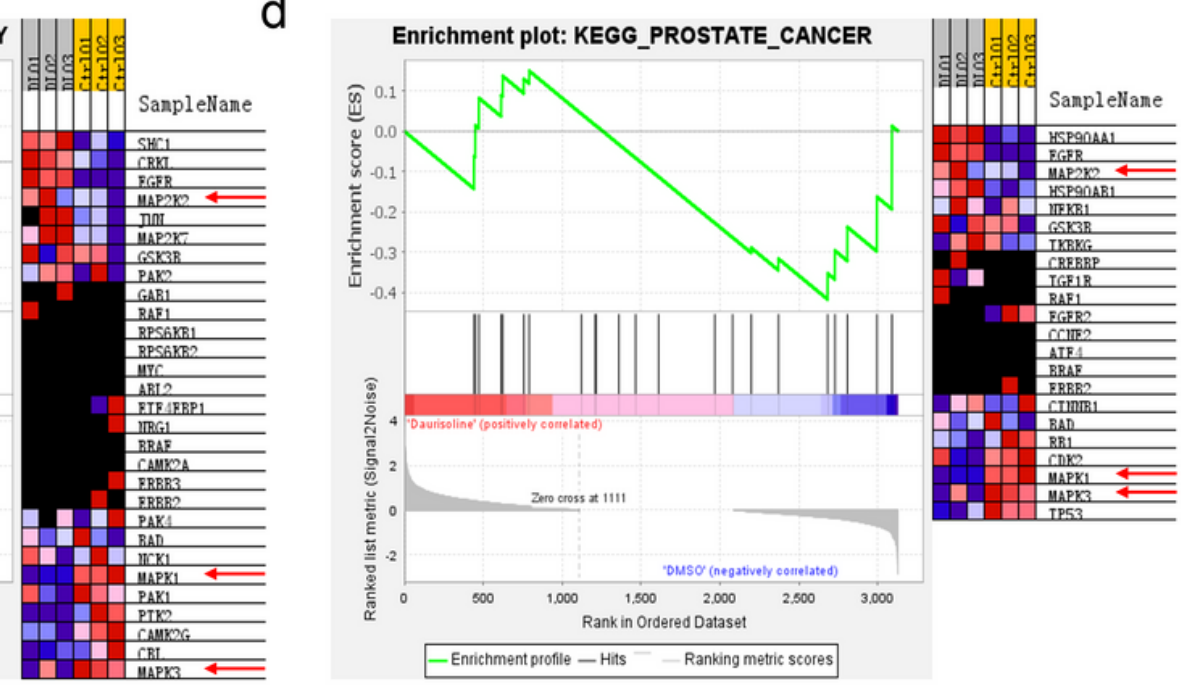

f

\begin{tabular}{|c|c|c|c|c|c|c|}
\hline \multirow[b]{2}{*}{ Daurisoline $(\mu \mathrm{M})$} & & & & & & \multirow[b]{3}{*}{ 44/42 KD } \\
\hline & 0 & 2.5 & 5 & 10 & 20 & \\
\hline -ERK1/2 T185/Y187 & $=$ & $\equiv$ & $=$ & $=$ & & \\
\hline t-ERK1/2 & $=$ & & $\equiv$ & $=$ & & 44/42 KD \\
\hline p-MEK1/2 & $\underline{-}$ & 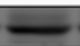 & ב & 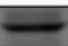 & 2 & $45 \mathrm{KD}$ \\
\hline t-MEK1/2 & $=$ & $\sqrt{3}$ & 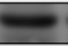 & $=$ & $=$ & $45 \mathrm{KD}$ \\
\hline GAPDH & & & & & 2 & $37 \mathrm{KD}$ \\
\hline
\end{tabular}


Daurisoline inhibits the MEK1/2-ERK1/2 signaling pathway in ESCC. a In vitro kinase assay of activeMEK1 and inactive ERK2. $b$ In vitro kinase assay of active-MEK2 and inactive ERK2. c,d The GSEA analysis of all proteins with varying expression levels in phosphoproteomic. The red arrow indicates MEK2 and ERK1/2. e,f Western blot of ERK and MEK expression levels in KYSE150 (left panel) and KYSE450 cells (right panel).

Fig. 5

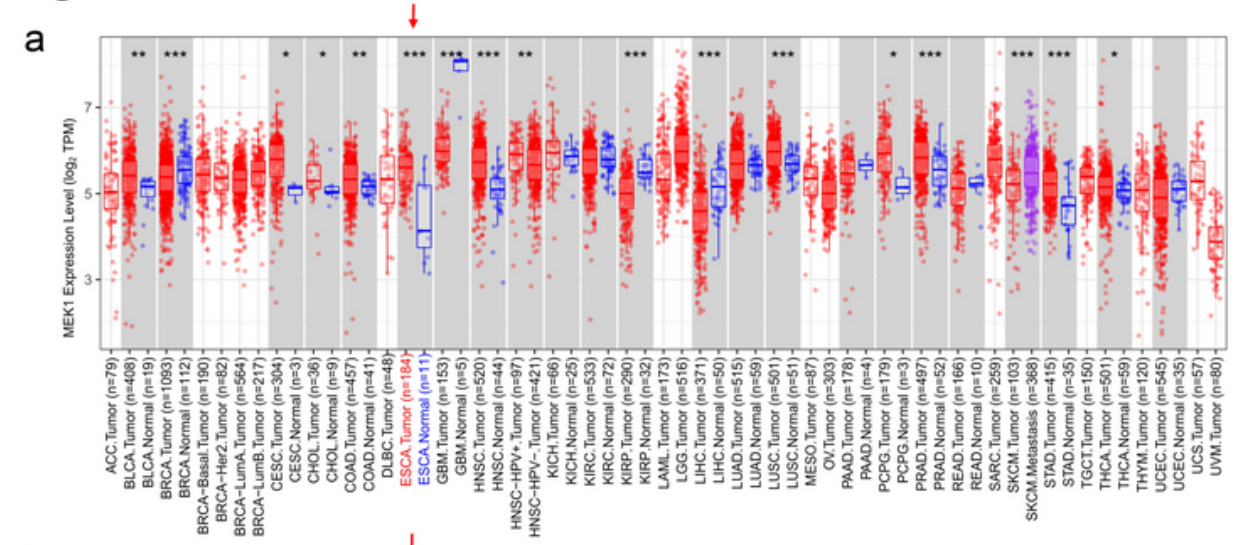

b

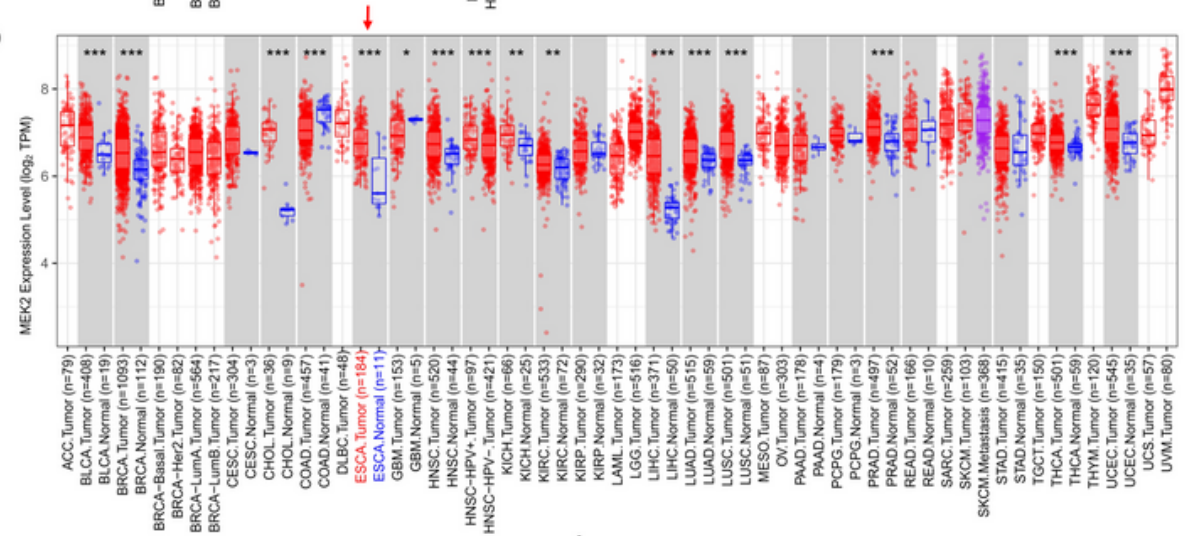

C

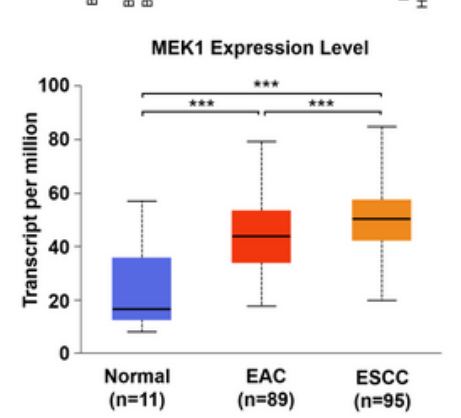

d

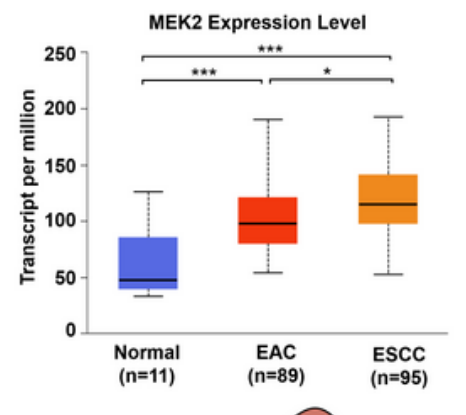

e
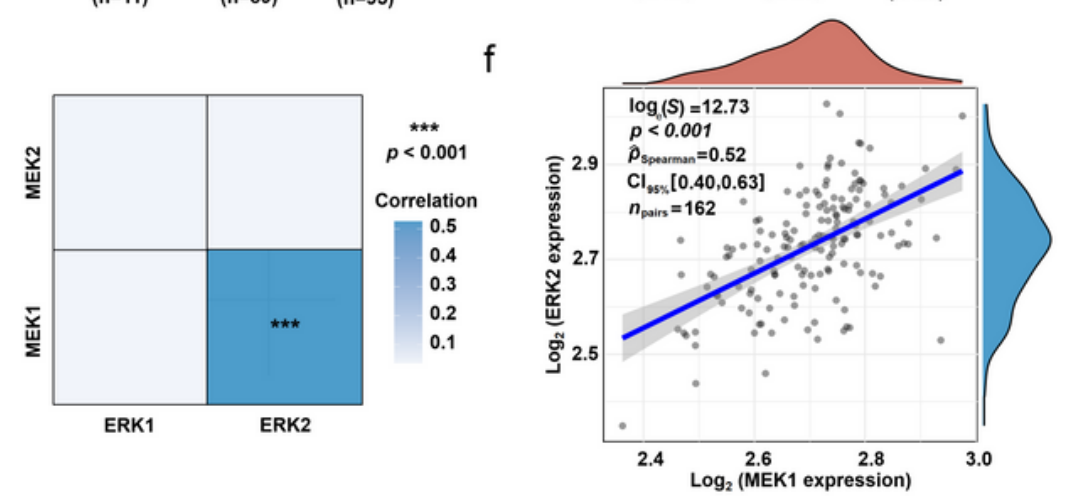

Figure 5 
MEK1/2 were significantly overexpressed in ESCC. a,b The expression of MEK1/2 in cancers of TCGA database. The red arrow indicates ESCA. c,d The expression of MEK1/2 in EAC and ESCC of TCGA database. e A heat map of the correlation between MEK1/2 and ERK1/2 of ESCC. The horizontal and vertical coordinates represent genes, and different colors represent correlation coefficients, and the darker the color represents the two stronger correlation. f Spearman correlation analysis of MEK1 expression and ERK2 expression. The horizontal axis in the figure represents the expression distribution of MEK1, and the ordinate is the expression distribution of ERK2. The density curve on the right represents the distribution trend of ERK2; the upper density curve represents the distribution trend of MEK1; In the top side the value represents the correlation $\mathrm{P}$ value, correlation coefficient and correlation calculation method. $\left({ }^{\star} P<0.05,{ }^{\star *} P<0.01,{ }^{\star \star *} P<0.001\right)$ 
Fig. 6
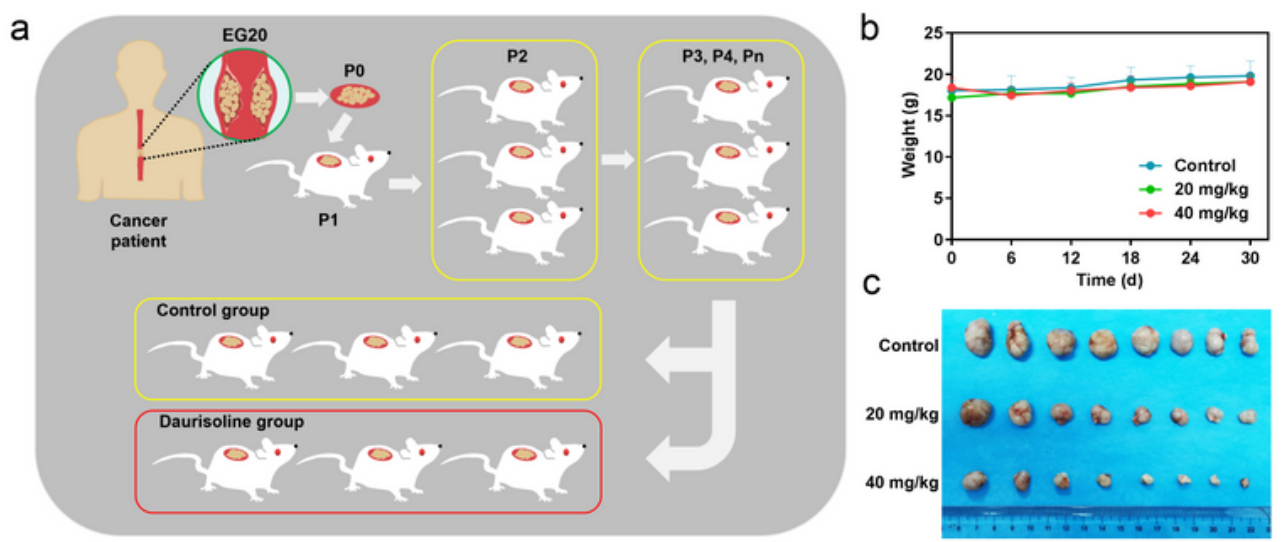

d
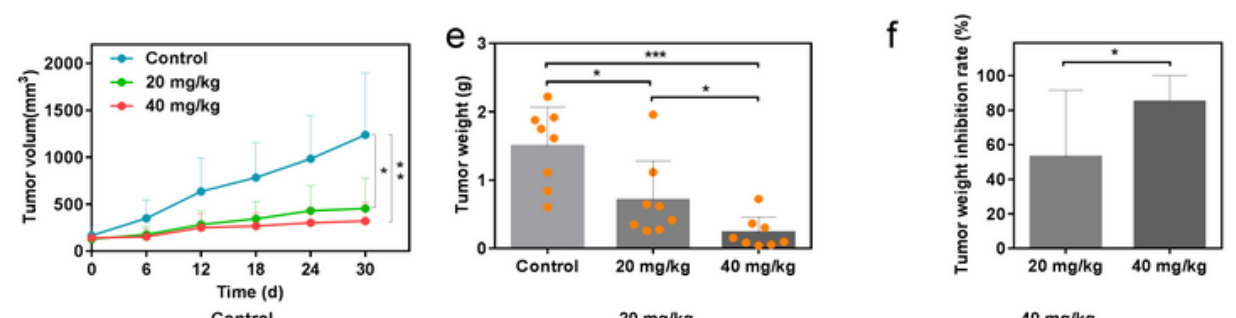

g
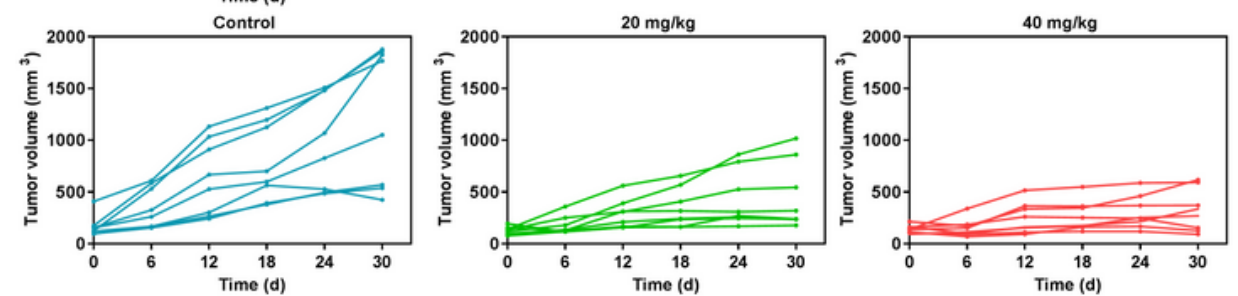

$\mathrm{h}$
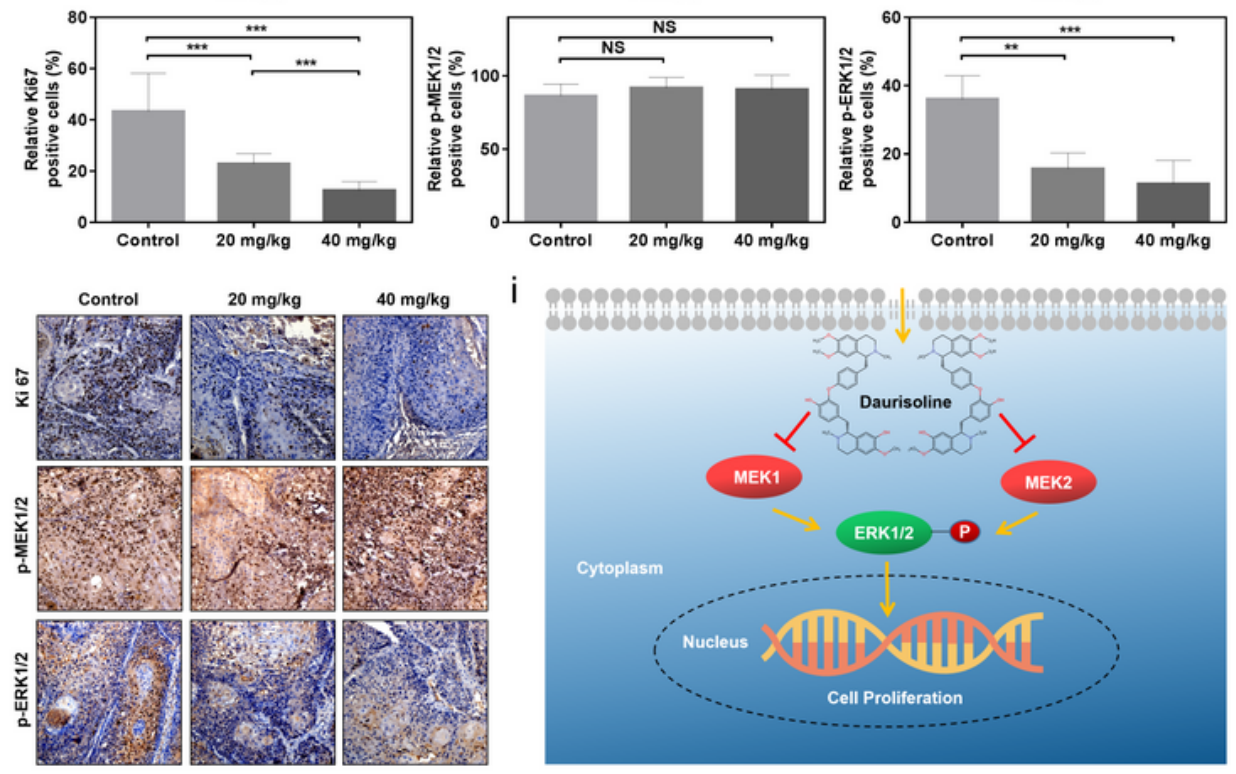

\section{Figure 6}

Daurisoline inhibit tumor growth of ESCC in vivo. a Schematic diagram of establishing PDX models of ESCC. $b$ Changes in body weight after treatment of the daurisoline. c Photographs of the EG20 tumor, $d$ changing curve of EG20 tumor volume, e tumor weight and $f$ tumor weight inhibition rate after treatment of daurisoline. g EG20 tumor volume changes of each mouse with different doses of daurisoline. $\mathrm{h}$ Immunohistochemical Staining of EG20 tumor tissue (bottom graph) and statistic graph (upper graph). i 
Schematic diagram of the signaling pathway about daurisoline inhibiting the growth of esophageal cancer. $\left({ }^{*} P<0.05,{ }^{*} \mathrm{P}<0.01,{ }^{* \star *} \mathrm{P}<0.001\right)$

\section{Supplementary Files}

This is a list of supplementary files associated with this preprint. Click to download.

- Supplementarymaterial.pdf 